\title{
LOS INICIOS DE LA PEQUEÑA EDAD DEL HIELO EN ESPAÑA. APORTACIONES DE LA CLIMATOLOGÍA HISTÓRICA AL CLIMA DEL SIGLO XIV
}

\author{
Mariano Barriendos \\ mbarriendos@ub.edu \\ Josep Barriendos \\ Graduado en Geografía \\ Departamento de Historia y Arqueología, \\ Universidad de Barcelona, \\ Montalegre 6, \\ 08001 Barcelona
}

\begin{abstract}
Resumen: El presente trabajo de climatología histórica pretende aportar los resultados obtenidos para España en fuentes documentales y bibliográficas para el siglo XIV. La crisis bajomedieval muestra una vertiente climática a pesar de una disponibilidad precaria de información por el contexto histórico en la Península Ibérica y la falta de instituciones administrativas con capacidad de generar grandes series documentales.

Los resultados ofrecen una visión preliminar de los comportamientos de la variabilidad climática propia de la Pequeña Edad del Hielo. Unas primeras oscilaciones de fuerte frecuencia de inundaciones y sequías entre 1310 y 1330 desencadenan fuertes crisis productivas e incluso sanitarias. Entre 1371 y 1380 se produce una oscilación de gran magnitud con episodios hidrometeorológicos extremos muy frecuentes. Sin duda, el siglo XIV con la presencia de estas oscilaciones abre un periodo de fuerte irregularidad hidrometeorológica que se prolonga hasta mediados de siglo XIX.
\end{abstract}

Palabras clave: Climatología histórica, Crisis bajomedieval, España, Pequeña Edad del Hielo, Riesgos climáticos. 


\title{
The beginning of little ice age in Spain. Contributions of historical climatology to $14^{\text {th }}$ Century climate
}

\begin{abstract}
Present project on historical climatology aims to provide the results obtained for Spain in documentary and bibliographic sources for the $14^{\text {th }}$ Century. The late medieval crisis shows a climatic dimension despite the precarious availability of information due to the historical context in the Iberian Peninsula and the lack of administrative institutions with capacity to generate large documentary series.

The results offer a preliminary vision of climatic variability patterns of the Little Ice Age. A first alteration of high frequency of floods and droughts between 1310 and 1330 triggered strong productive and even health crises. Between 1371 and 1380 there is other oscillation of great magnitude with very frequent extreme hydrometeorological events. Undoubtedly, $14^{\text {th }}$ century, with the presence of these oscillations, opened a period of strong hydrometeorological irregularity that lasted until the mid-19 $9^{\text {th }}$ century.
\end{abstract}

Keywords: Climatic Hazards, Historical Climatology, Little Ice Age, Low Middle Age Crisis, Spain.

\section{Introducción}

La climatología histórica en España es todavía una especialidad con un recorrido modesto, a pesar del potencial que ofrecen los archivos históricos españoles para la identificación y análisis de episodios climáticos y meteorológicos adversos. Existen diferentes retos para este tipo de investigación, y resulta interesante poder explorarlos. El contexto actual de cambio global y sus incertidumbres ambientales y socio-económicas abren una vía de investigación retrospectiva: la recuperación de información de situaciones climáticas anómalas del pasado, supone una acumulación nada desdeñable de conocimiento, tanto sobre los procesos naturales implicados como de los patrones de respuesta socio-económicos.

Siguiendo el propósito de explorar vías de desarrollo de la climatología histórica en España, este trabajo pretende realizar una evaluación general sobre las fuentes disponibles y un análisis exploratorio sobre el comportamiento del clima en el siglo XIV. Se trata de un periodo muy lejano en el tiempo, pero con paralelismos interesantes respecto a la actualidad. Los periodos de transición climática entre episodios climáticos de características diferentes, son los que acumulan mayor incertidumbre e irregularidad climática y meteorológica. Los primeros resultados de la investigación en climatología histórica centraban el análisis en comportamientos térmicos muy evidentes, con lo que el paleoclima reciente definió periodizaciones térmicas entr un Episodio Cálido 
Medieval y una Pequeña Edad del Hielo que ubicaba en la Edad Moderna desde mediados de siglo XVI a mediados de siglo XIX (entre otros, Lamb, 1972; Grove, 1988), con unos máximos de déficit térmico en el periodo del "Late Maunder Minimum" entre 1675 y 1715 (Pfister et al., 1994). Sin embargo, la proliferación de investigaciones, con diferentes proxy-data y con resoluciones espacio-temporales de mayor detalle, han permitido definir ya actualmente la Pequeña Edad del Hielo como un periodo de mayor extensión temporal, que se inicia en la Baja Edad Media (siglos XIII-XIV) de forma progresiva desde las latitudes más altas. Y la transición climática que lleva del Episodio Cálido Medieval hacia ese nuevo episodio entre, aproximadamente, 1250 y 1400, manifiesta ya un deterioro climático con la extensión de glaciares alpinos, mayor presencia de inviernos severos, dificultades en la navegación y mayor presencia de eventos hidrometeorológicos severos (entre otros, Pfister et al., 1996; Pfister et al., 1998; Ogilvie y Jónsson, 2001; Grove, 2001).

Se puede aceptar, pues, que el siglo XIV constituye una transición desde el Episodio Cálido Medieval a la Pequeña Edad del Hielo, mientras que en la actualidad el siglo XXI se caracteriza por una larga transición desde un episodio frío (Pequeña Edad del Hielo) hacia un calentamiento global en tendencia progresiva. En ambos casos, la variabilidad climática presenta una componente de anomalía porque supone un cambio de contexto desde una normalidad previa a una nueva normalidad con condiciones climáticas diferentes. Estos periodos de transición producen una serie de importantes impactos en los ecosistemas naturales y, en la vertiente social, en las actividades productivas, las infraestructuras, e incluso la salud pública de las poblaciones.

Además del interés por un periodo con el clima en transición, el siglo XIV presenta unas dificultades considerables en nuestro contexto histórico: es un territorio con amplias zonas en conflicto bélico con los reinos árabes y con algunas regiones en proceso de colonización poblacional y agraria. Por ello, es fácil suponer que se trata de un periodo en el que las administraciones están en proceso de institucionalización, y las redes de núcleos urbanos también se extienden y consolidan progresivamente. En esas circunstancias, la capacidad de generar fuentes documentales es muy precaria. Es un momento de creación y consolidación, tanto a nivel público como privado, de archivos y series documentales, por lo que su disponibilidad en aquellos momentos iniciales es limitado, discontinuo, dispar, y se ha conservado de forma precaria y fragmentada. Otro aspecto técnico también dificulta la generación y conservación de fondos documentales: en aquella época, los soportes escriptorios que se emplean están en transición desde el pergamino hacia la introducción progresiva del papel. La diferencia clave es que mientras se emplea el pergamino, su capacidad de reciclaje rápido en forma de palimpsestos impide la acumulación de información por el expurgado rápido que supone esta técnica. La introducción del papel, en cambio, permite ya una acumulación sucesiva de documentos en depósitos y archivos, existiendo la posibilidad de establecer series documentales cronológicas que son la base imprescindible para los estudios de reconstrucción climática. 
La disponibilidad precaria de información en fuentes documentales primarias repercute también en la producción historiográfica. Resulta obvio que los historiadores a su vez tengan dificultades para reconstruir en detalle todo tipo de procesos sociales y también, en consecuencia, los aspectos ambientales y climáticos para este periodo bajomedieval. Estas dificultades para disponer de una base de conocimiento de análisis fácil o cómodo son útiles para promover y poner a prueba nuevos planteamientos metodológicos y enfoques para el análisis de un periodo problemático. En este sentido, el presente artículo pretende revisar las metodologías aplicadas hasta ahora y proponer, teniendo en cuenta las informaciones disponibles para el siglo XIV, un procedimiento de análisis lo más eficaz posible. Esta aproximación pretende cubrir el territorio peninsular español poniendo en análisis integrado el conjunto de fenómenos hidrometeorológicos adversos y procesos de impacto relacionables con ellos. Para ello, se propone un apartado de análisis temporal para identificar las anomalías climáticas existentes, y un segundo apartado para estudiar las distribuciones espaciales de los fenómenos identificados, focalizando algún caso de estudio que resulte relevante.

\section{Estado de la cuestión en climatología histórica en España}

La investigación en el ámbito de la climatología histórica en España presenta algunas singularidades que resulta conveniente y útil poner de manifiesto. El potencial de investigación es ingente gracias al patrimonio documental conservado, por su magnitud y diversidad, y la cobertura temporal y geográfica que ofrece. Los procesos climáticos y ambientales en general que experimenta la Península Ibérica también justifican la investigación sobre el comportamiento de fenómenos adversos. Es especialmente interesante por su alta aplicabilidad a contextos actuales conocer el comportamiento de fenómenos hidrometeorológicos extremos, como sequías y lluvias torrenciales, por su incidencia en los recursos hídricos. Sin embargo, el desarrollo de la especialidad ha sido errático y su estudio pone de manifiesto la existencia de iniciativas muy meritorias pero esporádicas, inconexas, y con un escaso respaldo de las administraciones públicas ni las instituciones académicas (Barriendos, 1995, 1999, 2006).

Los inicios fueron muy prometedores con los trabajos de Manuel Rico Sinobas, médico y físico vallisoletano que en el marco del positivismo del siglo XIX inició la exploración de archivos históricos para la reconstrucción de comportamientos relevantes para la meteorología y la climatología. Sus trabajos son un referente por la precocidad mostrada en muchos aspectos, como el referido a las causas de la sequía en Almería y Murcia (Rico Sinobas, 1851). Pero su ejemplo no fructificó y durante decenios sólo se realizaron algunas recopilaciones de eventos por parte de historiadores locales o profesionales y que se prolongarían esporádicamente durante el siglo XX (entre otros, Bentabol, 1900; Blasco, 1959; Couchoud, 1965). 
El siglo XX no supone un avance para la especialidad en muchos decenios. El predominio de los planteamientos deterministas, con una fuerte componente política, centra los esfuerzos de recopilación en la utilización de la información para la explicación de sucesivos contextos y conflictos políticos y sociales, sin entrar en el conocimiento de los comportamientos climáticos por ellos mismos. Destacan en este enfoque autores como Ignacio Olagüe (Olagüe, 1951), y en especial José María Fontana Tarrats, que llegó a crear una base de datos con miles de registros sobre fenómenos meteorológicos, plagas y epidemias, que empleó en una serie de monografías mecanoscritas para diferentes reinos históricos de España. Sus trabajos de los años 60 y 70 del siglo XX confirman el potencial investigador del patrimonio documental español. Pocos años después, una primera publicación sobre paleoclima reciente del Instituto Nacional de Meteorología empleó en buena medida los materiales recopilados y catalogados por Fontana Tarrats (Font Tullot, 1988).

A finales de siglo XX se produce el desarrollo de la climatología histórica según las pautas investigadoras predominantes en el contexto europeo, concretándose en la presentación de tesis doctorales. Uno de los primeros trabajos se centra en las inundaciones en Baleares a partir de fuentes documentales e impresas (Grimalt, 1992). Poco después, otras dos tesis doctorales afrontan un estudio general sobre comportamiento climático durante la Edad Moderna a escala regional para Andalucía Oriental (Sánchez Rodrigo, 1994) y para Catalunya (Barriendos, 1994). Posteriormente, han proliferado estudios que empleando recopilaciones acotadas de información desarrollan índices climáticos anuales y mensuales para la identificación de la variabilidad climática (Martín Vide y Barriendos, 1995; Vicente-Serrano y Cuadrat, 2007, Rodrigo y Barriendos, 2008). En una etapa posterior se ha explorado también la posibilidad de combinar diferentes fuentes de información (multi-proxy approach) como los trabajos que integran fuentes documentales con registros paleohidrológicos (Thorndycraft et al., 2006) o con series dendroclimáticas (Tejedor et al., 2019). Por último, los recursos informáticos permiten un acceso mucho más detallado a las fuentes documentales con aplicación de herramientas de búsqueda de contenidos o formatos específicos, creando oportunidades para aplicar nuevas metodologías de reconstrucción climática y de análisis de sus repercusiones en los diferentes contextos sociales afectados (Gil-Guirado et al., 2019).

De forma muy reciente, la alarma social e incertidumbre producida por los potenciales impactos de un clima sometido a un calentamiento progresivo ha favorecido los estudios retrospectivos sobre eventos hidrometeorológicos extremos en periodos temporales amplios. En concreto, se ha impulsado un esfuerzo metodológico y material para la recopilación, catalogación y clasificación de eventos de inundación, causados básicamente por lluvias persistentes o torrenciales (Barriendos et al., 2014). Y se ha podido trabajar en la caracterización climática de estos eventos hidrometeorológicos a escala plurisecular (Barriendos et al., 2019). 
Además del trabajo en la dimensión de eventos extremos hidrometeorológicos que desarrolla la climatología histórica, actualmente también se está considerando la vertiente social de esa misma temática. Es decir, a partir de las mismas fuentes documentales y bibliográficas se estudia el fenómeno natural pero también los impactos, percepciones y respuestas de los diferentes colectivos sociales, desde instituciones públicas a particulares. Dos tesis doctorales en esta línea permiten ver con optimismo el futuro de la especialidad por la diversidad y profundización en los temas tratados, y que enlazan con el nuevo marco investigador de la ecología social a nivel internacional: la del geógrafo Salvador Gil-Guirado (2013) y de la ambientóloga Mar Satorras (2017). La producción científica ya empieza a dar resultados, encuadrados en el ámbito o especialidad de la ecología social (Gómez-Baggethun et al., 2012; Gil-Guirado et al., 2016).

\section{Fuentes y métodos aplicados en el presente trabajo}

\subsection{Fuentes de información para el estudio del clima del siglo XIV}

El siglo XIV tiene interés para su investigación climática por ser un periodo de transición desde el Episodio Cálido Medieval hacia la Pequeña Edad del Hielo. La crisis productiva y demográfica que se produce, ampliamente estudiada por los historiadores, es un indicio de algún tipo de crisis climática que desencadena o agrava esas dificultades sociales. Hay estudios clásicos que describen una situación con un patrón similar en diferentes ámbitos o contextos, lo que refuerza la idea de que intervienen factores globales en la misma (Seibt y Eberhard, 1992; Bois, 2001; Ruiz, 2008). Trabajos recientes han podido emplear incluso datos en alta resolución para ilustrar mejor los mecanismos de interrelación entre los aspectos productivos y demográficos que quedan comprometidos durante este periodo (Maltas, 2019).

El propio contexto de crisis no facilita la generación y conservación de fuentes documentales, y en consecuencia su análisis e interpretación por parte de los historiadores. Por otro lado, el siglo anterior había registrado un importante avance en el proceso de Reconquista, por lo que amplios territorios estaban en pleno proceso de colonización y de consolidación de nuevas instituciones. Durante el siglo XIV, este proceso de avance general quedó detenido en la frontera del Reino de Granada, y se reemplazó por una serie de conflictos entre reinos cristianos, prueba de que la situación presentaba alguna anomalía que afectaba sistemas productivos e institucionales: las guerras CastellanoAragonesas de 1288-1291, 1296-1304 y 1356-1369.

El contexto histórico es suficientemente complejo como para no proporcionar un patrimonio documental que permitiría un trabajo de reconstrucción climática óptimo: las fuentes proporcionan información esporádica, dispersa, discontinua. No se dispondrá de series documentales continuas y homogéneas, y suficientemente diversas, hasta bien entrado el siglo XVI. 
El presente trabajo se adapta a estas circunstancias de disponibilidad, y ha recopilado información en una gama amplia y diversa de fuentes bibliográficas y documentales. Entre otras, historiografía local, fuentes documentales privadas, crónicas reales, y la incipiente documentación administrativa de instituciones de gobierno municipal y eclesiástico. Una fuente singular que se ha empleado por su amplia cobertura espaciotemporal es la base de datos recopilada por José María Fontana Tarrats, conservada en los archivos de la Agencia Estatal de Meteorología.

Buena parte de los materiales disponibles se habían recopilado en diferentes proyectos pero sin poder ser analizados ya que su baja densidad impedía la generación de índices mensuales o estacionales. Por ello, estos datos tienen una utilización apenas existente y se mantienen inéditos, salvo para trabajos recientes sobre inundaciones en la costa mediterránea (Barriendos et al., 2019).

\subsection{Distribución de la información disponible}

La información recopilada para el siglo XIV se concreta en 320 casos o unidades de información. Esto representa una densidad relativamente baja de informaciones, poco más de 3 por año. Cuando la información lo permite por su nivel de detalle temporal, se han propuesto agrupaciones de casos que responden a eventos meteorológicos o situaciones adversas que pueden singularizarse y producir un registro de episodio. El resultado de la aplicación de estos criterios preliminares, siendo conscientes de la escasa densidad de información y las dificultades para un análisis consistente por la considerable distancia temporal, ofrece un total de 211 episodios.

Una primera organización de la información debe intentar evitar unidades políticas o administrativas que en siglos posteriores experimentarán cambios sustanciales y frecuentes. Ha resultado eficaz la aplicación de un criterio fisiográfico, recurriendo a las cuencas hidrográficas que se distribuyen de forma homogénea y completa por todo el territorio. De este modo, la localización de las informaciones se realiza según la organización actual de demarcaciones hidrográficas (Instituto Geográfico Nacional, 2019). La distribución de casos (Tabla 1) ofrece un resultado dispar. Hay cuencas que no ofrecen información ( 0 casos) porque son territorios en pleno proceso de colonización o porque todavía son reinos musulmanes. El sesgo que también se observa se produce por un mayor trabajo aplicado en las cuencas de la vertiente mediterránea por haber aplicado más esfuerzo investigador en aquella zona (Tabla 2).

La localización de las poblaciones que disponen de casos registrados (Figura 1) confirma el pronunciado sesgo hacia la vertiente mediterránea, aunque su distribución territorial es mínimamente representativa de casi todas las cuencas y permite la detección y valoración de las anomalías climáticas más relevantes. 
Tabla 1. Distribución de los casos recopilados por cuencas hidrográficas

\begin{tabular}{|l|l|c|}
\hline Cuenca & Denominación & No casos \\
\hline CIG & Cuencas Internas de Galicia & 1 \\
\hline NOR & Cuenca Norte (Miño) & 3 \\
\hline COC & Cuencas del Cantábrico Occidental & 1 \\
\hline COR & Cuencas del Cantábrico Oriental & 9 \\
\hline CHD & Cuenca Hidrográfica del Duero & 1 \\
\hline CHT & Cuenca Hidrográfica del Tajo & 0 \\
\hline CGN & Cuenca Hidrográfica del Guadiana & 0 \\
\hline CAA & Cuenca Atlántica Andaluza (Guadalete+Barbate) & 9 \\
\hline CAA & Cuenca Atlántica Andaluza (Tinto+Odiel+Piedras) & 0 \\
\hline CGQ & Cuenca Hidrográfica del Guadalquivir & 15 \\
\hline CMA & Cuenca Mediterránea Andaluza & 34 \\
\hline CHS & Cuenca Hidrográfica del Segura & 98 \\
\hline CHJ & Cuenca Hidrográfica del Júcar & 117 \\
\hline CHE & Cuenca Hidrográfica del Ebro & 4 \\
\hline CIC & Cuencas Internas de Cataluña & 1 \\
\hline BAL & Cuencas Internas de Baleares & 0 \\
\hline FRA & Cuencas de Catalunya Nord & 17 \\
\hline TOT & Localizaciones generales o indeterminadas & \\
\hline
\end{tabular}

Tabla 2. Distribución de los casos recopilados por vertientes hidrográficas

\begin{tabular}{|l|c|c|}
\hline Vertiente peninsular & No de casos & Porcentaje (\%) \\
\hline Vertiente Atlántica & 25 & $7,8 \%$ \\
\hline Vertiente Mediterránea & 269 & $84,1 \%$ \\
\hline Localizaciones indeterminadas & 26 & $8,1 \%$ \\
\hline
\end{tabular}






Figura 1. Distribución espacial de las poblaciones con información disponible para el siglo XIV. Número de poblaciones con registros en cada demarcación hidrográfica.

La organización por episodios muestra, por lo menos, una distribución temporal muy consistente. Si agrupamos los 211 episodios por quinquenios a lo largo del siglo XIV, todos ellos contienen episodios. En promedio, cada quinquenio tiene 11 episodios, y sus valores extremos van desde los 3 episodios (quinquenio 1316-1320) a 19 episodios (quinquenio 1376-1380).

\subsection{Detalles metodológicos sobre la generación de la base de datos}

La información recopilada se ha organizado según unos criterios no aplicados previamente. La investigación en periodos históricos tan alejados ofrece una densidad de información muy baja y no parecía viable un esfuerzo de análisis como el del presente trabajo. La organización, catalogación y clasificación de la información exige unas ciertas singularidades. Un primer elemento a considerar es que la información predominante se refiere a situaciones extraordinarias y a los impactos que puede producir en la actividad humana. No hay registros que refieren a situaciones de normalidad y muchos menos a condiciones favorables de clima o de obtención de cosechas. 
En consecuencia, la propuesta metodológica renuncia a un seguimiento del clima en sus condiciones de normalidad y variabilidad mediante los habituales sistemas de índices numéricos a diferente resolución temporal y que son aptos para la reconstrucción climática de siglos posteriores (por ejemplo, Glaser et al., 1999; Camuffo et al., 2010). Tratar sólo eventos meteoclimáticos extremos es la solución posibilista ante la ausencia de otras informaciones en el estado presente de la investigación en fuentes documentales y bibliográficas. De hecho, las características generales del clima mediterráneo en la Península Ibérica describen un importante protagonismo de los comportamientos de fuerte variabilidad temporal con frecuentes episodios extraordinarios. Los testimonios históricos sólo ponen énfasis en las condiciones climáticas relevantes, por las características del fenómeno natural, y por las graves repercusiones sociales sufridas, con impactos directos en infraestructuras y cosechas, así como impactos diferidos en diversos ámbitos, incluyendo la salud pública.

La catalogación de la información se realiza priorizando los detalles básicos que permiten datar la información y ubicarla con la mayor precisión posible. Se llega a una datación diaria, que se mantiene en formato de calendario original, sin forzar ninguna transformación entre sistemas de calendario juliano y gregoriano. Las localizaciones geográficas presentan gran diversidad de detalle: las mayoritarias refieren poblaciones, que pueden adscribirse a la estructura actual de términos municipales. Un número menor de registros son muy generales, refiriendo zonas o regiones completas que están afectadas por el fenómeno. Estas informaciones menos definidas, habitualmente también con una datación poco detallada, se catalogan para su uso como soporte o contexto, pero difícilmente pueden entrar en un análisis como el que se podría aplicar en informaciones de siglos posteriores.

Las referencias a cursos fluviales son quizás el elemento más útil para la localización de la información. Son elementos naturales que apenas experimentan alteraciones importantes a escala histórica. Por otro lado, las denominaciones toponímicas de los cursos fluviales se mantienen en el tiempo, y sus posibles variaciones son fácilmente identificables.

La clasificación de la información se ajusta a los formatos y condiciones en las que puede obtenerse. Por supuesto, hay una importante diversidad, desde registros muy específicos a descripciones generales e imprecisas, por lo que el esfuerzo se centra en la posibilidad de aglutinar el máximo de registros con unas categorías mínimas comunes muy generales.

Las unidades de información son el elemento más simple que se puede catalogar y clasificar para su análisis posterior. Cuando durante el proceso de catalogación se detectan diferentes casos con coherencia temática y temporal, se agrupan y se crea un episodio. La utilidad de estas agrupaciones proviene de la fuerte variabilidad existente en el registro de casos, de tal manera que por falta o exceso de disponibilidad de información, por causas ajenas a la propia dinámica meteorológica, si sólo se consideraran los casos registrados, se podrían producir fuertes sesgos en algunos análisis. 
En cuanto a las categorías que pueden llegar a identificarse y aplicarse a los casos registrados, se ha decidido proponer una tabla muy simplificada pero que puede dar representación a todos los casos. Por supuesto, este desarrollo es una propuesta ajustada a las limitaciones del material disponible para el siglo XIV. Es de esperar que, en futuras investigaciones, el estudio de periodos más ricos y diversos permita generar propuestas de clasificación mucho más cercanas a los procedimientos actuales. Las situaciones adversas se organizan en 6 grupos y 13 subgrupos, cuyas definiciones se detallan a continuación:

Grupo 1: ERE (Extraordinary Rainfall Event). Evento de lluvia extraordinaria.

Subgrupo 1: FF (Fluvial Flood).

Crecidas o desbordamientos en cursos fluviales por lluvias extraordinarias.

Subgrupo 2: PF (Pluvial Flood).

Inundaciones por drenaje deficiente en eventos de lluvias extraordinarias.

Subgrupo 3: PR (Persistent Rainfall).

Eventos de lluvia de duración extraordinaria.

Grupo 2: DRO (Drought). Sequías.

Subgrupo 4: DR (Drought).

Sequías por déficit de precipitaciones con diversos impactos en el medio natural y agrario.

Grupo 3: CAE (Convective Activity Event). Evento de actividad convectiva.

Subgrupo 5: SS (Sea Storm).

Temporales de mar con afectación en la navegación o infraestructuras litorales.

Subgrupo 6: HE (Hail Event).

Tempestades de granizo y pedrisco con afectaciones en ámbito rural o urbano.

Subgrupo 7: ES (Electric Storm).

Tempestades con afectaciones por actividad eléxtrica extraordinaria.

Grupo 4: NTA (Negative Thermic Anomaly). Anomalía térmica negativa.

Subgrupo 8: CW (Cold Wave).

Olas de frío con heladas extraordinarias, fuera de calendario o en zonas no habituadas.

Subgrupo 9: US (Unusual Snowfall).

Nevadas extraordinarias, de gran magnitud o en zonas o calendario no habituales. 
Grupo 5: PTA (Positive Thermic Anomaly). Anomalía térmica positiva.

Subgrupo 10: HW (Heat Wave).

Olas de calor fuera de calendario habitual o de magnitud extraordinaria.

Grupo 6: SIE (Social Impact Event). Evento de impacto social.

Subgrupo 11: EE (Epidemic Event).

Brote epidémico por infecciones de afectación humana.

Subgrupo 12: PE (Plague Event).

Enfermedades o afectaciones en cultivos o ganado.

Subgrupo 13: FS (Food Shortage).

Situación de escasez de productos alimentarios básicos; alteración de precios de mercado.

La propuesta de clasificación se organiza en cinco grupos que definen fenómenos naturales, mientras un sexto grupo aglutina procesos de adversidad manifestados por la sociedad, pero cuyo origen no queda explícitamente identificado. La indefinición que se produce por parte de las fuentes directas contemporáneas y por las fuentes bibliográficas indirectas impide una asociación rápida entre fenómenos naturales y situaciones de impacto social. Por ello, es preferible mantener ambas dimensiones, natural y social, separadas en grupos propios.

Resulta habitual encontrar situaciones de impacto social con términos genéricos sin una explicación causal definida. No se pueden atribuir o interpretar los fenómenos naturales cuando cualquiera de ellos o una combinación de varios de ellos pueden ser los causantes. Por ejemplo, una esterilidad en la producción agraria o una crisis productiva general de una sociedad puede deberse a factores ambientales y climáticos, pero también puede deberse a procesos sociales ajenos o alejados de condicionantes ambientales (guerras y conflictos civiles, actos delictivos, revueltas y protestas, accidente, etc...). Por ello, hemos optado por segregar las informaciones sobre dificultades en la producción que no especifiquen la causa, dejándolas en un grupo de eventos de impacto social. De este modo, no se producen interpretaciones erróneas, y queda pendiente de futuras investigaciones la posibilidad de identificar correctamente los fenómenos que se producen en aquel momento y su naturaleza.

\section{Análisis de los resultados}

\subsection{Descripción general de los grupos y subgrupos identificados}

Los casos de eventos adversos, una vez clasificados, muestran una distribución con fenómenos preponderantes y fenómenos minoritarios (Tabla 3). 
Tabla 3. Distribución de los casos por grupos

\begin{tabular}{|l|c|c|}
\hline Grupos & $N^{\circ}$ Casos & Porcentaje \\
\hline ERE & 163 & $51 \%$ \\
\hline CAE & 22 & $7 \%$ \\
\hline DRO & 61 & $19 \%$ \\
\hline NTA & 11 & $3 \%$ \\
\hline PTA & 6 & $2 \%$ \\
\hline SIE & 57 & $18 \%$ \\
\hline
\end{tabular}

Los fenómenos naturales preponderantes son los de carácter hidrometeorológico. Las lluvias excesivas representan el $51 \%$ de los casos registrados, y la sequía casi un 20\%. En consecuencia, para el siglo XIV por lo menos, se puede considerar que el comportamiento meteorológico adverso más característico en el clima peninsular está relacionado con los excesos o déficits pluviométricos. La actividad convectiva queda algo más distante, y las anomalías térmicas, positivas y negativas, alcanzan el 5\%. El registro de impactos sociales se mantiene proporcionalmente en segundo plano con un 18\%, pero ya indica que las anomalías ambientales dejan de forma frecuente situaciones de impacto social, ya sea de forma directa o inducida (Tabla 3).

Como se ha explicado en capítulos anteriores, se ha propuesto dividir los grupos en subgrupos que permiten especificar y concretar más la tipología de la afectación del fenómeno analizado. El grupo con una mayor cantidad de casos, y en el que las subdivisiones aportan más matices, es el de los episodios de lluvia extraordinaria (ERE), cuyos subgrupos permiten diferenciar entre los tipos de episodios de lluvia que se pueden producir. Dentro de este grupo predomina claramente el subgrupo de inundaciones fluviales (FF) que con 145 casos representa un 89\% de los casos del grupo ERE y un 45\% de los casos totales (Tabla 4). De esta manera se sitúa como el tipo de episodios adversos predominantes para el siglo XIV. También dentro del grupo de los ERE se encuentran aquellas inundaciones de carácter pluvial (PF), diferenciables de las anteriores por tener una proporción mucho menor, y similar a la de los episodios de lluvias de larga duración (PR). Estos dos subgrupos se encuentran muy alejados en cantidad de casos a los de las inundaciones fluviales, representando solo un 11\% del total del grupo de los ERE y sumando 18 casos entre ambos (6\% del total) para el siglo XIV (Tabla 4).

El siguiente grupo, que merece una mención para comentar sus subdivisiones, es el que corresponde a los eventos que tienen un impacto social (SIE) porque con más de 50 casos representa el 18\% de la cantidad total de los casos (Tabla 4). Este grupo, del mismo modo que el grupo de los ERE, se encuentra dividido en 3 subgrupos pero en este caso se trata de tipos de fenómenos poco relacionados entre sí. En primer lugar, destaca la escasez frumentaria (FS) correspondiente a tres cuartos del total de casos de 
su grupo (SIE), un porcentaje del 75\% que equivale a un 13\% de los casos totales del periodo completo. Para este siglo, el siguiente subgrupo de los SIE en importancia corresponde con el de las epidemias (EE) (19\% del total de casos del grupo y un 3\% del total global), seguido del subgrupo de las plagas (PE) que, con 1\% del total y solo 3 casos, se sitúa como el fenómeno con una menor presencia en el siglo XIV (Tabla 4).

Tabla 4. Distribución de casos por subgrupos

\begin{tabular}{|l|c|c|c|}
\hline \multicolumn{4}{|c|}{ DISTRIBUCIÓN DE CASOS POR SUBGRUPOS } \\
\hline \multirow{2}{*}{ Erupos } & Sub-grupos & $N^{\circ}$ casos & Porcentaje \\
\cline { 2 - 4 } & PR & 10 & $3 \%$ \\
\cline { 2 - 4 } & FF & 145 & $45 \%$ \\
\hline \multirow{2}{*}{ DRO } & PF & 8 & $3 \%$ \\
\hline CAE & SS & 10 & $19 \%$ \\
\cline { 2 - 4 } & HE & 6 & $3 \%$ \\
\cline { 2 - 4 } & ES & 6 & $2 \%$ \\
\hline \multirow{3}{*}{ NTA } & CW & 7 & $2 \%$ \\
\cline { 2 - 4 } & US & 4 & $1 \%$ \\
\hline PTA & HW & 6 & $2 \%$ \\
\hline \multirow{3}{*}{ SIE } & EE & 11 & $3 \%$ \\
\cline { 2 - 4 } & PE & 3 & $13 \%$ \\
\cline { 2 - 4 } & FS & 43 & \\
\hline \multirow{3}{*}{} & & & $13 \%$ \\
\hline
\end{tabular}

El segundo grupo en cantidad de casos es el de las sequías (DRO), del mismo modo que también lo es su único subgrupo (DR). Este grupo representa alrededor de una quinta parte del número total de casos para el siglo XIV, con un porcentaje del 19\% y un total de 61 casos. Para el siglo analizado, algunos de los casos de escasez frumentaria se encuentran visiblemente relacionados o son consecuencia directa de las sequías. Si se combinan los casos del subgrupo de escasez alimentaria (FS) con los del grupo de las sequías (DRO), encontramos que se acerca a un tercio de los casos totales producidos en el periodo, con más de 100 casos y representando el 32\% del total (Tabla 4).

Los fenómenos vinculados a la actividad convectiva (CAE) y a las anomalías térmicas (NTA+PTA), tienen una presencia muy reducida. La actividad convectiva registra 22 casos, un $7 \%$ del total de casos registrados. Las anomalías térmicas todavía tienen menor incidencia: en conjunto, sólo registran 17 casos, el 5\% del total de casos registrados (Tabla 4). 


\subsection{Análisis temporal. Frecuencias}

El comportamiento de los eventos meteoclimáticos adversos durante el siglo XIV no permite un análisis eficiente. Ya se ha comentado anteriormente que la baja densidad de información disponible no da facilidades para detectar anomalías o estudiar en detalle episodios singulares. Por otro lado, el periodo de un siglo bajo análisis no es una escala de trabajo que en climatología histórica sea muy habitual por las limitaciones que supone. En todo caso, es una prueba suficiente para un trabajo exploratorio.

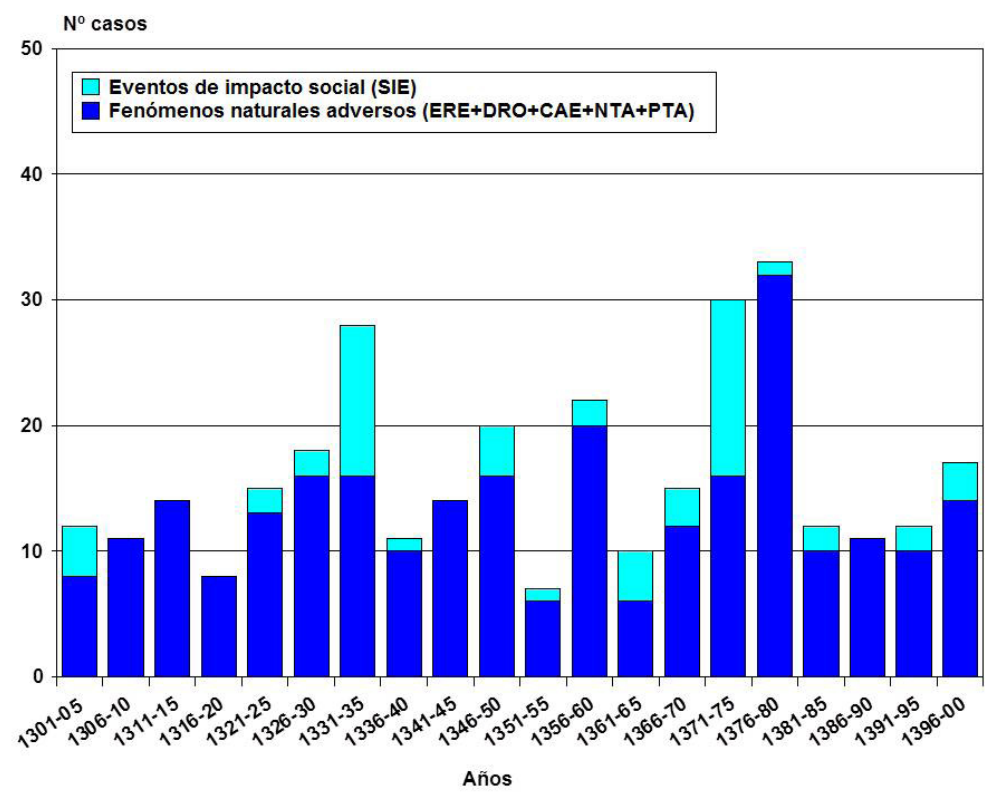

Figura 2. Distribución por quinquenios del número de casos de situaciones adversas.

Fuente: Elaboración propia.

El periodo del siglo XIV puede manifestar las anomalías que llevan del deterioro del Episodio Cálido Medieval a unas primeras manifestaciones de lo que será siglos después un comportamiento irregular y poco favorable para la actividad humana, la Pequeña Edad del Hielo. A pesar de ello, no se perciben cambios abruptos o comportamientos de tendencia significativos. A la vista de los resultados generales (Figura 2), aparecen unas oscilaciones en la frecuencia de las situaciones adversas, tal como se detectan en siglos posteriores en las mismas zonas de estudio (Barriendos, 1996-1997). Los valores de frecuencia inician el siglo XIV con valores inferiores a la media (16 casos por quinquenio). Los eventos o episodios registrados son de carácter natural, y apenas aparecen registros asociados a impactos sociales. Entre 1321 y 1335, en cambio, se 
detecta una oscilación de incremento moderado de este tipo de eventos, y una respuesta de impacto social, especialmente acusada en el quinquenio 1331-1335. Los quinquenios posteriores muestran unas frecuencias bajas, inferiores a la media del siglo XIV, salvo dos periodos de frecuencia alta, 1346-1350 y 1356-1360. En este periodo central del siglo, es remarcable la presencia de registros sobre impacto social que acompaña los fenómenos adversos. El mismo patrón se observa ya hasta finales de siglo.

Sin embargo, destaca el periodo 1371-1380 con la presencia de alrededor del doble de casos y con una importante presencia de impactos sociales. Se puede considerar este periodo como una segunda oscilación de anomalía climática, más breve que la primera, pero más severa. A pesar de la escasa información disponible, la ocurrencia de dos oscilaciones importantes dentro de un periodo inferior a un siglo no es muy habitual por lo documentado en detalle entre los siglos XVI y XIX (Barriendos, 1996-1997), por lo que se justifica la relevancia del siglo XIV, justificada por la presencia de dos periodos con una marcada presencia de eventos de impacto social (1331-1335 y 1371-1375).

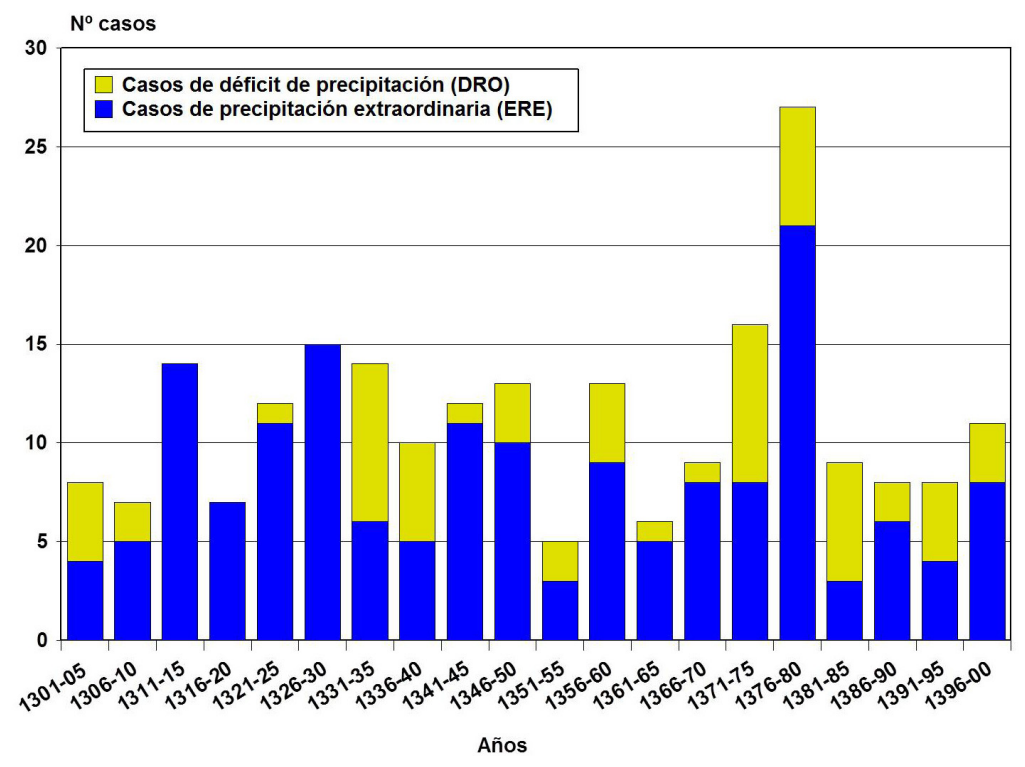

Figura 3. Distribución por quinquenios de los casos de lluvia extraordinaria (ERE) y déficits de precipitación (DRO).

Fuente: Elaboración propia.

Si se tratan en detalles los fenómenos adversos más habituales, lluvias extraordinarias y sequías, se puede percibir la configuración interna de las oscilaciones antes identificadas (Figura 3). La distribución general de los quinquenios de frecuencia baja o media ofrece una relación equilibrada entre situaciones de precipitación extraordinaria (ERE) 
y de sequía (DRO). La primera oscilación (1321-1335) muestra una configuración compleja, con un fuerte incremento de los casos tipo ERE en la primera parte o fase ascendente de la oscilación. Después los casos ERE se reducen rápidamente, pero aparecen eventos de tipo DRO con una incidencia no experimentada en lo que iba de siglo. Esta situación compleja y contrastada, pudo ser resultado de un incremento de la irregularidad climática y, sin duda, puede tener una estrecha relación con los graves impactos sociales referidos en este periodo, como las hambrunas generalizadas del año 1333.

La oscilación de 1371-1380 presenta un comportamiento más acusado de irregularidad pluviométrica. Durante todo el periodo anómalo, el incremento principal lo registran los eventos de exceso de precipitación (ERE) pero las sequías (DRO) acompañan este crecimiento. Se trata de una oscilación en la que se incrementa simultáneamente la frecuencia de excesos y déficits de precipitación. Son situaciones evidentemente, muy difíciles para una sociedad basada en actividades primarias y dependientes mayoritariamente de una agricultura de secano. La sucesión de eventos de signo contrario producen una importante alteración en los rendimientos agrícolas y en otras actividades vinculadas al recurso hídrico. No es fácil encontrar referentes similares en la variabilidad climática reciente. Quizás una situación similar, aunque más prolongada en el tiempo se produjo con la denominada Oscilación Maldà, entre los años 1760 y 1800 (Barriendos, 1996-1997; Barriendos y Llasat, 2003).

\subsection{Análisis espacial de periodos anómalos}

La identificación de periodos con fenómenos adversos supone un avance en el conocimiento de la variabilidad del clima en el siglo XIV. A pesar de la baja densidad de información, su distribución temporal muestra algunas oscilaciones breves pero significativas por lo que suponen de impacto para la sociedad de la época. Con la finalidad de obtener un resultado más completo con la información disponible, se han seleccionado los años con mayor número de registros singulares dentro de las oscilaciones de 1321-1335 y 1371-1380, para su representación cartográfica. De este modo, se puede visualizar la información y detectar incongruencias que exigirían un replanteamiento metodológico en la selección y utilización de la información. Por otro lado, se pretende averiguar si la extensión y diversidad de fenómenos puede ser tan relevante como para explicar las consecuencias adversas e impactos demográficos y económicos que el inicio de la Pequeña Edad del Hielo deja en la Península Ibérica.

El primer caso de estudio corresponde al primer año en el que los impactos por adversidades meteoclimáticas son muy severas: 1333 (Figura 4). Las crónicas de Barcelona lo refieren como "Lo mal any primer" (El primer mal año). Y ciertamente las adversidades registradas presentan una distribución general por la Península Ibérica: se produce sequía en Galicia, Castilla, y Aragón, así como escasez de alimentos en Cataluña. Pero dentro de la última, también aparecen diferentes localizaciones que expli- 
citan problemas por sequía. Se produce alguna inundación en zonas litorales y fríos severos en la Meseta Norte. La identificación de los problemas y su localización no dejan demasiada duda para atribuir a una fuerte sequía los impactos en la sociedad que se producirán especialmente en la vertiente mediterránea, centrados en una disminución acusada en el aprovisionamiento de alimentos básicos.

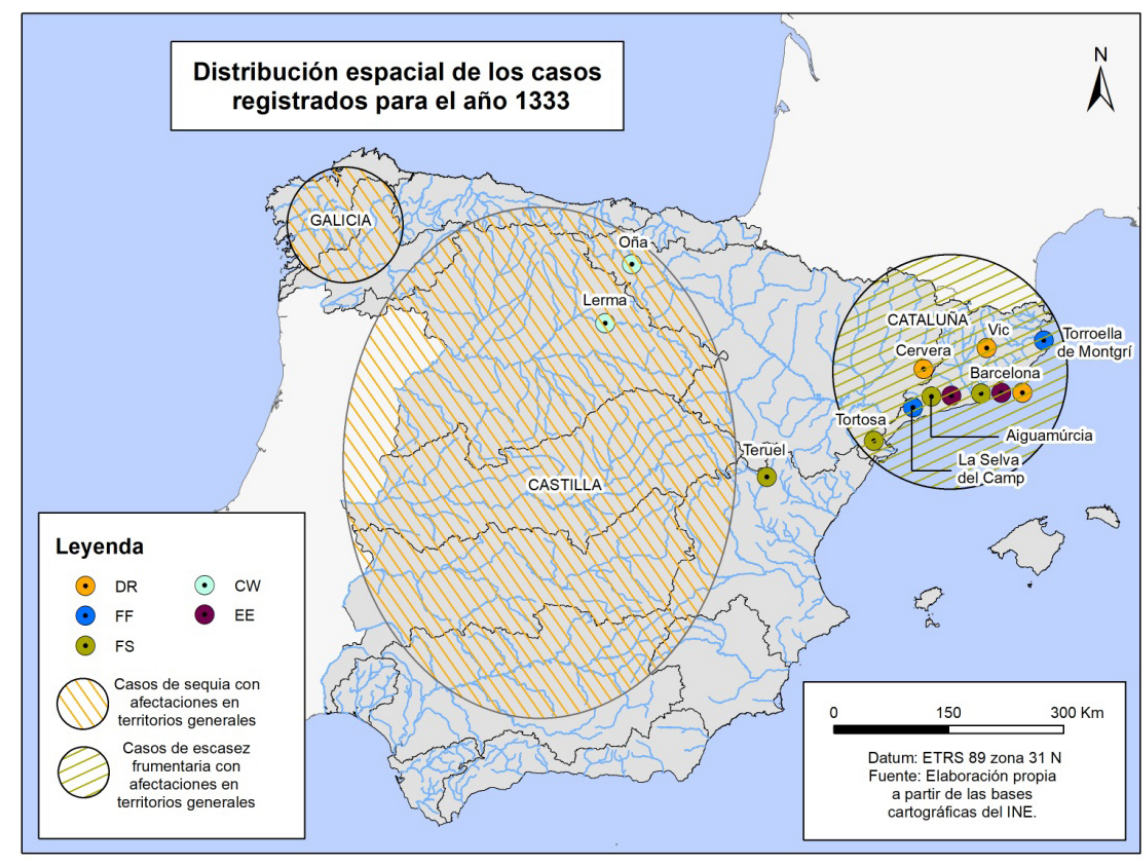

Figura 4. Distribución espacial de los casos registrados para el año 1333.

Otro caso se produce ya dentro de la oscilación severa y compleja de 1371-1380. El año 1374 registra abundantes casos, todos ellos de sequía y escasez de alimentos básicos, y se distribuye de forma muy coherente en los antiguos reinos de la Corona de Aragón: Aragón, Cataluña, Baleares y Valencia (Figura 5). Una sequía probablemente severa por los problemas de aprovisionamiento que se producen, y que al tratarse de una zona amplia y continua, debía incrementar los problemas para los envíos de suministros de ayuda entre regiones, ya que todas estaban sometidas a las mismas condiciones adversas. Pero en el clima mediterráneo, las sequías no son incompatibles con lluvias extraordinarias, de modo que se producen desbordamientos fluviales en cuencas amplias, como el Ebro en Tortosa, y el Guadalquivir en Sevilla.

Para concluir la descripción del comportamiento espacial de las anomalías es interesante centrarse en la parte final del decenio de fuerte irregularidad, en su periodo 


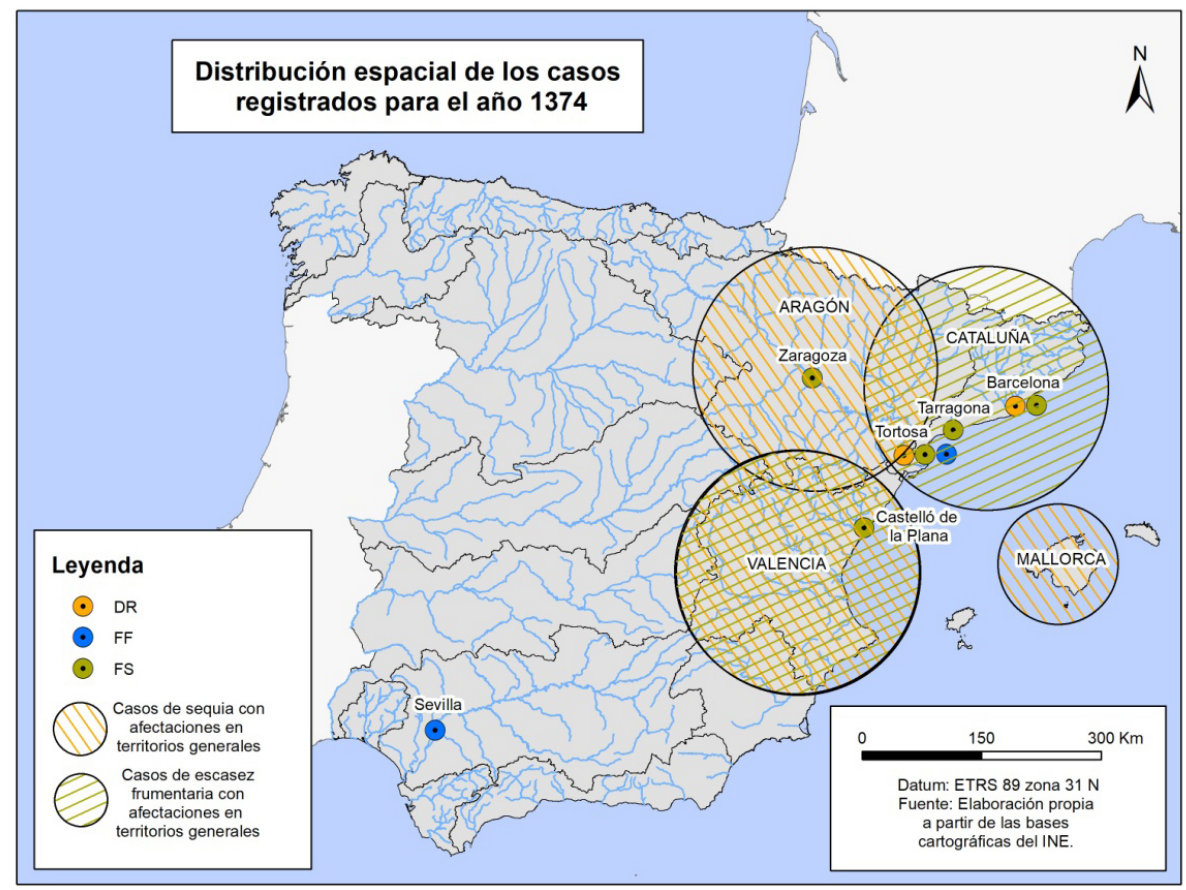

Figura 5. Distribución espacial de los casos registrados para el año 1374.

álgido (Figura 6). Los años 1375 y 1376 se caracterizan por fuertes sequías y escasez de alimentos en Aragón, Cataluña y Valencia, tanto en referencias territoriales generales como en poblaciones concretas.

Sin embargo, los años 1379 y 1380 acumulan registros sobre una situación totalmente opuesta que provoca numerosas inundaciones por lluvias extraordinarias en una amplia porción de territorio (Figura 7). De hecho, la cartografía de aquel periodo muestra un año 1379 de lluvias extraordinarias en el valle del Ebro en su tramo central y en su afluente pirenaico, el Segre. Aunque el evento ya es de singular extensión porque se producen también inundaciones en la cuenca del Segura y lluvias persistentes en la cabecera del Turia (Teruel).

El año 1380 supone un reforzamiento de estas condiciones de precipitación extraordinaria con una extensión máxima del episodio: Se repiten los desbordamientos en el Ebro, el Segre y el Segura, pero también se producen noticias sobre lluvias persistentes en Castilla e inundaciones en la desembocadura del Ebro y en la cuenca del Ter en Cataluña. Los perjuicios e impactos diferidos de una situación tan general y persistente, a falta de mayores detalles en las fuentes disponibles, cabe suponer que serían muy graves. 


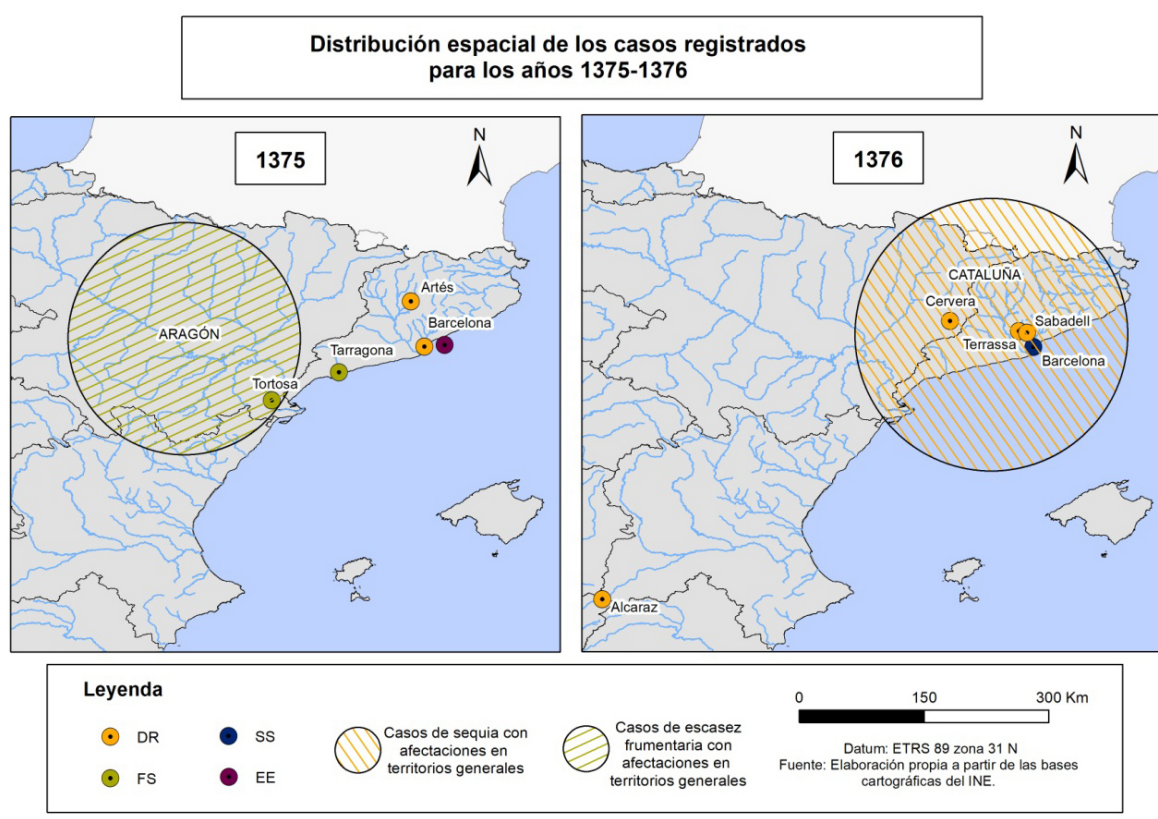

Figura 6. Distribución espacial de los casos registrados para el año 1375-1376.

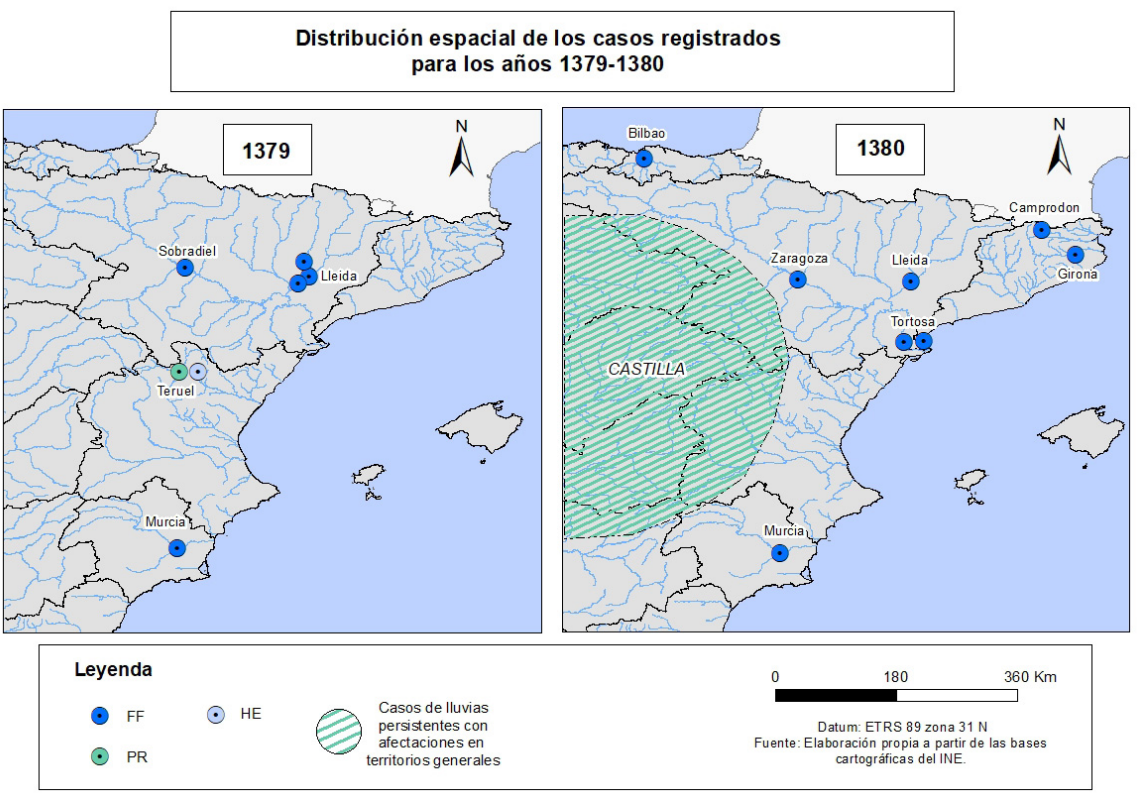

Figura 7. Distribución espacial de los casos registrados para los años 1379-1380. 


\section{Discusión de los resultados}

La disponibilidad de información en las fuentes históricas no permite unos resultados óptimos para el periodo estudiado. Sin embargo, las metodologías desarrolladas de catalogación y clasificación de los registros permiten un análisis compatible al que se desarrollaría en investigaciones de periodos históricos posteriores con mayor disponibilidad de información. Se ha optado por unas categorías muy simples, pero de concepción amplia. Así en trabajos para periodos posteriores es muy difícil que aparezca la necesidad de desarrollar alguna nueva. Sin embargo, la compartimentación abierta en subgrupos sí que permite, a medida que la información aparezca con más calidad de detalle, poder generar una mayor variedad de subcategorías más adaptadas a entornos complejos y más densamente poblados como ocurre en los siglos recientes.

A pesar de la información disponible escasa, su representación cartográfica es un procedimiento que permite visualizar la totalidad de los registros con lo que el análisis de las situaciones adversas es mucho más eficiente que empleando sistemas de cuantificación por índices u otros procedimientos gráficos. Para el estudio de casos específicos o anomalías de corta duración, la localización de las informaciones en una base cartográfica temática es un apoyo muy útil para el análisis de los fenómenos estudiados, y permite su comparación con las cartografías de eventos que se generan actualmente a partir de registros instrumentales. El empleo de programario especializado para la edición cartográfica directamente desde bases de datos (Sistemas de Información Geográfica) representa un avance significativo en las capacidades de análisis paleoclimático. La información creciente sólo puede analizarse ya con soportes informáticos y su representación cartográfica aproxima los resultados a las cartografías temáticas que s eproducen en la actualidad para situaciones similares.

Respecto a los fenómenos registrados, los más frecuentes son los desbordamientos por lluvias extraordinarias, mientras que las inundaciones pluviales o las lluvias persistentes apenas aparecen. Quizás en contextos históricos diferentes, esta proporción puede modificarse. Pero en el siglo XIV es evidente que la población tenía que exponerse y ubicarse próximos a los cursos fluviales para disponer del recurso hídrico. Ello implica una vulnerabilidad intrínseca al riesgo por inundaciones fluviales, mucho menor para otros riesgos climáticos, se irá prolongando en el tiempo mientras las capacidades constructivas y el desarrollo tecnológico no permitan superar la servitud de la exposición al mismo.

El escaso número de registros vinculados a anomalías térmicas, a pesar de estudiar un periodo que se considera el inicio de un episodio climático caracterizado por los déficits térmicos, parece indicar dos posibles factores: es posible que la población no tuviera una vulnerabilidad notable a las anomalías de este tipo. En segundo lugar, es posible que la Pequeña Edad del Hielo no representara en las latitudes mediterráneas un impacto térmico negativo como el que produjo en latitudes altas y en Centroeuropa. 
No obstante, la importante cantidad de registros genéricos que simplemente se describen como "Tiempo estéril" o "Cosecha estéril", quizás esconde detrás algún factor climático todavía no identificado o un conjunto de factores más complejos. Sólo futuras investigaciones pueden ir aclarando estas incertidumbres.

Respecto a los eventos vinculados a impactos sociales, destaca la gran cantidad de registros de escasez frumentaria. Aunque no es posible todavía vincularlos a determinadas adversidades climáticas, su representación cartográfica ha permitido definir amplias extensiones de afectación, que ayudarán a identificar y comprender los procesos ambientales que puede haber detrás. La combinación de diferentes subcategorías en los mapas también permite comprender la evolución de los episodios complejos. Por ejemplo, en 1333 se puede observar sucesivamente como se inician casos de sequía prolongada desde el año anterior, ésta se generaliza, se producen fuertes carestías, y finalmente crisis epidémicas en la costa mediterránea.

La dimensión temporal del análisis es muy modesta, pero la complejidad de la variabilidad climática del siglo XIV ofrece manifestaciones relevantes. Dos oscilaciones climáticas breves, pero de fuerte magnitud y complejidad, dentro de un solo siglo, permiten entender que fue un contexto climático y ambiental muy duro para la sociedad de la época. De estas oscilaciones destaca su naturaleza básicamente hidrometeorológica: comportamientos irregulares en el registro de precipitaciones, con protagonistas destacados en las lluvias torrenciales causantes de inundaciones fluviales y las sequías. Por lo que conocemos del comportamiento durante la Pequeña Edad del Hielo, se producen oscilaciones climáticas de similares características, más prolongadas, de unos 40 años de duración, pero al menos en la vertiente mediterránea española entre los siglos XVI y XIX sólo se perciben tres eventos o fases de este tipo, por lo que la presencia de dos oscilaciones, aunque breves, dentro del mismo siglo XIV puede significar que se estuvo produciendo una anomalía de características relevantes.

\section{Conclusiones}

Los comportamientos hidrometeorológicos extraordinarios se registran de forma prolija debido a la afectación que producen en la sociedad. La evidente vulnerabilidad ante el exceso o déficit de recurso hídrico será una constante desde el inicio de la Pequeña Edad del Hielo.

Se percibe un predominio de situaciones adversas vinculadas a los eventos hidrometeorológicos extraordinarios, tanto por exceso (lluvias torrenciales causantes de inundaciones), como por déficit (sequías).

Existe una evidente organización temporal en la frecuencia de las situaciones adversas: dos oscilaciones con combinaciones complejas de diferentes tipos de eventos. 
La primera entre 1321 y 1335 , se inicia con frecuentes lluvias con inundaciones y termina con sequías. La segunda, entre 1371 y 1380, tiene un comportamiento más complejo, con un incremento simultáneo de inundaciones y sequías. Estos eventos de fuerte irregularidad pluviométrica tienen escasos referentes, como la Oscilación Maldà (17601800), pero también son las situaciones que apuntan para un futuro próximo los modelos climáticos de precipitación.

Los resultados obtenidos en este articulo permiten apreciar que el sistema de clasificación propuesto soporta los registros generados para un periodo breve y precario como el siglo XIV. Para reconstrucciones climáticas más eficaces, es aconsejable extender la investigación a periodos prolongados que contengan mucha más información de detalle.

\section{Bibliografía}

Barriendos, M. (1995). La recerca en climatologia històrica. Un debat necessari per al desenvolupament correcte de l'especialitat a l'àmbit de la Mediterrània Occidental, Treballs de la Societat Catalana de Geografia 10 (40), pp. 19-32.

Barriendos, M. (1996-1997). El clima histórico de Catalunya (siglos XIV-XIX). Fuentes, métodos y primeros resultados, Revista de Geografía 30-31, pp. 69-96.

Barriendos, M. (1999). La climatología histórica en el marco de la antigua Monarquía Hispana. Scripta Nova 3 (53), http://www.ub.es/geocrit/nova.htm.

Barriendos, M.; Llasat, M.C. (2003). The Case of the 'Maldá' Anomaly in the Western Mediterranean Basin (AD 1760-1800): An Example of a Strong Climatic Variability, Climatic Change 61, pp. 191-216. DOI: http://dx.doi.org/10.1023/a:1026327613698.

Barriendos, M. (2006). La climatología histórica en el contexto universitario español. Pedralbes. Revista d'Història Moderna 26, pp. 41-63.

Barriendos, M.; Ruiz-Bellet, J.L.; Tuset, J.; Mazón, J.; Balasch, J.C.; Pino, D.; Ayala, J.L. (2014). The 'Prediflood' database of historical floods in Catalonia (NE Iberian Peninsula) AD 1035-2013, and its potential applications in flood analysis. Hydrology and Earth System Sciences 18, pp. 1-17. DOI: http://dx.doi.org/10.5194/hess-18-1-2014.

Barriendos, M.; Gil-Guirado, S.; Pino, D.; Tuset, J.; Pérez-Morales, A.; Alberola, A.; Costa, J.; Balasch, J.C.; Castelltort, X.; Mazón, J.; Ruiz-Bellet, J.L. (2019). Climatic and social factors behind the Spanish Mediterranean flood event chronologies from documentary sources (14th-20th centuries). Global and Planetary Change 182 (102997). DOI: http://dx.doi.org/10.1016/j.gloplacha.2019.102997.

Bentabol, H. (1900). Las aguas de España y Portugal. Madrid: Vda. e Hijos de M. Tello, $2^{\underline{a}}$ ed.

Blasco, J. (1959). Las avenidas del Ebro. Crecidas verdaderamente extraordinarias, 1261-1959. Zaragoza: Librería General.

Bois, G. (2001). La Gran depresión medieval: siglos XIV-XV: el precedente de una crisis sistémica. Valencia, Universidad de Valencia. 
Camuffo, D.; Bertolin, C.; Barriendos, M.; Dominguez-Castro, F.; Cocheo, C.; Enzi, S.; Sghedoni, M.; della Valle, A.; Garnier, E.; Alcoforado, M.J.; Xoplaki, E.; Luterbacher, J.; Diodato, N.; Maugeri, M.; Nunes, F.; Rodriguez, R. (2010). 500-year temperature reconstruction in the Mediterranean Basin by means of documentary data and instrumental observations. Climatic Change 101, pp. 169-199. DOI: http:// dx.doi.org/10.1007/s10584-010-9815-8.

Couchoud, R. (1965). Hidrología histórica del Segura, Efemérides hidrológica y fervorosa recopilada y escrita por Dr. R. Couchoud. Madrid: Centro de Estudios Hidrográficos.

Font Tullot, I. (1988). Historia del clima de España. Cambios climáticos y sus causas. Madrid: Instituto Nacional de Meteorología.

Gil-Guirado, S. (2013). Reconstrucción climática histórica y análisis evolutivo de la vulnerabilidad y adaptación a las sequías e inundaciones en la cuenca del Segura (España) y en la cuenca del río Mendoza (Argentina). Tesis doctoral. Universidad de Murcia, Departamento de Geografía Física, Humana y Análisis Regional.

Gil-Guirado, S., Espín-Sánchez, J.A. \& Prieto, M.D.R. (2016). Can we learn from the past? Four hundred years of changes in adaptation to floods and droughts. Measuring the vulnerability in two Hispanic cities. ClimaticCchange 139(2), pp. 183-200.

Gil-Guirado, S., Gómez-Navarro, J.J. \& Montávez, J.P. (2019). The weather behind words-new methodologies for integrated hydrometeorological reconstruction through documentary sources. Climate of the Past 15(4), pp. 1303-1325.

Glaser, R.; Brázdil, R.; Pfister, C.; Dobrovolny, P.; Barriendos, M.; Bokwa, A.; Camuffo, D.; Kotyza, O.; Limanowka, D.; Racz, L.; Rodrigo, F.S. (1999). Seasonal Temperature and Precipitation fluctuations in Selected parts of Europe during the Sixteenth Century. Climatic Change 43, pp. 169-200.

Gómez-Baggethun, E., Reyes-García, V., Olsson, P. \& Montes, C. (2012). Traditional ecological knowledge and community resilience to environmental extremes: A case study in Doñana, SW Spain. Global Environmental Change 22(3), pp. 640-650.

Grimalt, M. (1992). Geografia del risc a Mallorca. Les Inundacions. Palma de Mallorca: Institut d'Estudis Baleàrics.

Grove, J.M. (2001). The initiation of the 'Little Ice Age' in regions round the North Atlantic. Climatic Change 48, pp. 53-82.

Instituto Geográfico Nacional (2019). https://miteco.gob.es/es/cartografia-y-sig/ide/descargas/ agua/demarcaciones-hidrograficas-phc-2015-2021.aspx.

Lamb, H.H. (1972). The cold Little Ice Age climate of about 1550 to 1800. En: Lamb, H.H. (Ed.) Climate: present, past and future. London: Methuen, p. 107.

Maltas, J. (2019). Caresties, fams i crisis de mortalitat a Catalunya: 1283-1351. Anàlisi d'indicadors i reconstrucció dels cicles econòmics i demogràfics. Tesis doctoral. Departamento de Historia, Universidad de Lleida, 998 p. http://hdl.handle.net/10803/667511.

Martín Vide, J. y Barriendos, M. (1995). The use of rogation ceremony records in climatic reconstruction: a case study from Catalonia (Spain). Climatic Change 30, pp. 201-221.

Ogilvie, A.E.J.; Jónsson, T. (2001). 'Little Ice Age' research: a perspective from Iceland. Climatic Change 48, pp. 9-52. 
Olagüe, I. (1951). La decadencia española, Madrid: Mayfé, 4 vols.

Pfister, C.; Kington, J.; Kleinlogel, G.; Schüle, H.; Siffert, E. (1994). High resolution spatio-temporal reconstructions of past climate from direct meteorological observations and proxy data. En: Frenzel, B. (Ed.): Climatic trends and anomalies in Europe 1675-1715. High resolution spatio-temporal reconstructions from direct meteorological observations and proxy data. Methods and results. Jena: Gustav Fischer Verlag, pp. 329-375.

Pfister, C.; Schwarz-Zanetti, G.; Wegmann, M. (1996). Winter severity in Europe: the fourteenth century, Climatic Change 34, pp. 91-108.

Pfister, C.; Luterbacher, J.; Schwarz-Zanetti, G.; Wegmann, M. (1998): Winter air temperature variations in western Europe during the Early and High Middle Ages (AD 750-1300), The Holocene 8(5), pp. 535-552.

Rico Sinobas, M. (1851). Memoria sobre las causas meteorologico físicas que producen las constantes sequías de Murcia y Almería, señalando los medios de atenuar sus efectos. Madrid: D.S. Compagni.

Rodrigo, F.S.; Barriendos, M. (2008). Reconstruction of seasonal and annual rainfall variability in the Iberian Peninsula (16th-20th Centuries) from documentary data. Global and Planetary Changes 63, pp. 243-257.

Ruiz, T.F. (2008). Las crisis medievales: 1300-1474. Madrid: Crítica.

Satorras, M. (2017). Adaptation before Anthropogenic Climate Change: A Historial Perspective on Adaptation to Droughts in Terrassa (1600-1870s, NE Spain). Tesis doctoral. ICTA, Universitat Autònoma de Barcelona.

Seibt, F.; Eberhard, W. (1992). Europa 1400: la crisis de la baja edad media. Madrid: Crítica.

Tejedor, E.; de Luis, M.; Barriendos, M.; Cuadrat, J.M.; Luterbacher, J.; Saz, M.A. (2019). Rogation ceremonies: a key to understanding past drought variability in northeastern Spain since 1650. Climate of the Past 15, pp. 1-18. DOI: http://dx.doi.org/10.5194/cp-15-1-2019.

Thorndycraft, V.; Barriendos, M.; Benito, G.; Rico, M.; Casas, A. (2006). The catastrophic floods of A.D.1617 in Catalonia (NE Spain) and their climatic context. Hydrological Sciences Journal 51 (5), pp. 899-912.

Vicente-Serrano, S.M.; Cuadrat, J.M. (2007). North Atlantic oscillation control of droughts in northeast Spain: evaluation since 1600 A.D. Climatic Change 85, pp. 357-379. 
